\title{
Inguinal lymph node dissection for penile cancer: a contemporary review
}

\author{
Jiasian Teh ${ }^{1,2,3,4}$, Catriona Duncan ${ }^{1,2}$, Liang $\mathbf{Q u}^{2}$, Glen Guerra ${ }^{3,4,5}$, Vignesh Narasimhan ${ }^{3,4,5}$, \\ Toan Pham ${ }^{3,4,5}$, Nathan Lawrentschuk ${ }^{1,3,5,6}$
}

${ }^{1}$ Department of Surgery, Austin Hospital, University of Melbourne, Melbourne, Victoria, Australia; ${ }^{2}$ Young Urology Researchers Organisation (YURO), Melbourne, Victoria, Australia; ${ }^{3}$ Division of Cancer Surgery, ${ }^{4}$ Division of Cancer Research, Peter MacCallum Centre, Melbourne, Victoria, Australia; ${ }^{5}$ Sir Peter MacCallum Department of Oncology, University of Melbourne, Melbourne, Victoria, Australia; ${ }^{6}$ Olivia Newton-John Cancer Research Institute, Austin Hospital, Melbourne, Victoria, Australia

Contributions: (I) Conception and design: J Teh; (II) Administrative support: J Teh; (III) Provision of study materials or patients: J Teh; (IV) Collection and assembly of data: J Teh, C Duncan; (V) Data analysis and interpretation: J Teh, C Duncan, L Qu; (VI) Manuscript writing: All authors; (VII) Final approval of manuscript: All authors.

Correspondence to: Dr. Jiasian Teh. Department of Urology, Austin Health, Level 8, Harold Stokes Building, 145 Studley Road, Heidelberg, VIC 3084, Australia. Email: jiasian@gmail.com.

\begin{abstract}
Penile cancer is an uncommon disease associated with significant psychological and physical morbidity. Penile cancer has an expectable pattern of spread in a stepwise fashion, from inguinal to pelvic lymph nodes (PLN) then distant spread. Patients with penile cancer have variable survival, with patients with a low burden of nodal metastatic disease having lasting survival with surgical management, however patients with a large amount of locoregional metastatic disease having a worse prognosis. The current management options for patients with metastatic lymph node disease in penile cancer aims to reduce the morbidity associated with radical inguinal lymph node (ILN) surgery with appropriate risk stratification to optimise oncological control of the disease. This article describes current challenges in managing the inguinal region in patients with penile squamous cell carcinoma (SCC).
\end{abstract}

Keywords: Penile cancer; inguinal lymph node metastasis (ILN metastasis); surgical oncology

Submitted Mar 01, 2019. Accepted for publication Aug 26, 2019.

doi: $10.21037 /$ tau.2019.08.37

View this article at: http://dx.doi.org/10.21037/tau.2019.08.37

\section{Introduction}

Penile cancer is a rare disease associated with significant morbidity and mortality $(1,2)$. Whilst it is rare in developed countries, with an incidence of around 1/100,000 men, in parts of Africa and South America, penile cancer can account for approximately $10-20 \%$ of all male malignancies $(3,4)$. Squamous cell carcinomas (SCC) account for over $95 \%$ of all penile cancers, with several subtypes of varying prognostic significance including basaloid, papillary, warty, verrucous, sarcomatoid, adenosquamous and mixed (5). Risk factors for penile cancer include poor hygiene, circumcision status, human papilloma virus (HPV) infection, phimosis, human immunodeficiency virus (HIV) and smoking (4).
Additionally, the diagnosis and subsequent treatment of penile cancer is often delayed, largely due to patients failing to seek help, from a lack of awareness or the associated stigmata with diseases of the genital organs (6). Consequently, patients often present with locally advanced tumours (7). The mainstay of treatment for localised disease is surgery, with neoadjuvant cisplatin-based combination chemotherapy used for high-risk and locally advanced disease.

The most clinically significant prognostic factor for patients with penile cancer is the involvement of locoregional lymph nodes (8). Recurrence free and overall survival are largely dependent on inguinal lymph node (ILN) disease burden, with the 5-year survival for patients 
with a single involved inguinal node approximately $80 \%$, compared with $0-12 \%$ if deeper pelvic lymph nodes (PLN) are involved $(9,10)$. Lymph node metastasis in penile cancer occurs in a consistent pattern, first involving the ILNs and subsequently the PLN in keeping with the route of anatomical drainage. The first LNs to be involved are the 'sentinel' lymph nodes, which are found in the superior medial zone of the inguinal region, followed by the central inguinal zones (11). Current management of lymph node metastasis in penile cancer aims to decrease the morbidity related to traditional surgical approaches of radical inguinal lymphadenectomy, without compromising oncological outcomes. Patients with low-volume lymphatic disease can usually achieve cure with surgery alone, however patients with extensive locoregional lymph node involvement are less likely to reach long-term survival without multimodal management and potentially radical and morbid surgery (12).

Over the last decade, advances have been made in the diagnosis, stratification and treatment of men with penile SCC. Whilst increased use of organ-preserving surgery and minimally invasive LN staging has reduced the morbidity of the disease, the survival rates of patients with penile cancer with lymph node metastasis has not significantly changed in the USA or Europe since the 1990s (13). This potentially may be due in part to the underutilisation of proven therapy, in particular ILN dissection (ILND) rather than a lack of advanced treatments (14). Additionally, penile SCC belongs to a group of SCCs associated with HPV infection, including anal, cervical and oropharyngeal SCC, with parallels in treatment drawn from these other cancer sites.

In this review, we aim to present contemporary trends in the management of inguinal lymph metastasis in penile cancer, surgical techniques relating to inguinal and PLN dissection, and the role of chemotherapy and radiotherapy. We also aim to explore the potential use of emerging immunotherapeutic options for the treatment of patients with SCC of the penis.

\section{Indications for lymphadenectomy}

\section{Impalpable ILNs}

A key issue in the management of patients with penile cancer is that occult metastasis cannot be detected with physical examination or non-invasive methods, such as imaging with ultrasound, CT or MRI, alone. The management strategies for patients with clinically normal ILNs are: surveillance, invasive nodal staging or radical lymphadenectomy.

Surveillance of regional LNs is associated with the risk of disease recurrence developing in the future from undetected micro-metastatic disease, as patients with clinically nodenegative disease at the time of diagnosis ( $\mathrm{cN} 0)$ having a risk of micro-metastases to ILNs of approximately $25 \%$ (15). This risk can be further stratified according to histopathological features of the primary tumour, and the management of this patient group should be directed by pathological risk factors including local stage, grade and lymphovascular invasion, with the risk of micro-metastatic disease in low and intermediate risk disease of between $17-50 \%(16,17)$. Early ILND in cN0 patients offers increased long-term survival compared to delayed lymphadenectomy, with patient survival over $90 \%$ with early lymphadenectomy and less than $40 \%$ with lymphadenectomy for patients with regional recurrence $(18,19)$. Patients being considered for surveillance should be counselled of this potential risk, with surveillance being recommended only in patients with pTis/pTa tumours, if they are low risk G1pT1 tumours, and be undertaken only in patients who will be compliant with surveillance $(20,21)$. Imaging of the inguinal region plays a limited role in detecting micro-metastatic disease, however may be considered in patients in whom examination is limited or unreliable due to body habitus.

The surgical management of ILN metastasis requires thorough anatomical knowledge of the region. The ILNs are a collection of nodes draining the lower limb and groin. The nodal tissue superior to the fascia lata is considered superficial and nodal tissue, and below fascial lata considered deep. The superficial lymphatics drain the prepuce and the skin of the shaft to the ILNs. The glans and the deep penile structures are drained by separate lymphatic channels to the lymph nodes in the femoral triangle (22). The inguinal nodes are associated with the saphenous vein and femoral vessels located within the femoral triangle, demarcated by the inguinal ligament superiorly, the adductor longus muscle medially and the sartorius muscle laterally. Daseler et al. (23) divided the inguinal region into 5 zones according to their relationship to the surrounding veins. Of note, nodal metastases most commonly occur in zone $\mathrm{V}$, and Cloquet's node is in the central zone within the fossa ovalis. The extent of dissection and which nodal groups are taken is a significant consideration in the management of penile cancer, with the possible curative resection of any involved inguinal nodes providing long term survival benefit. However, this needs to be balanced with the significant morbidity of ILND. 
There are 2 invasive diagnostic modalities that are currently recommended in patients with high-risk primary penile cancer (>pT1G2 and/or presence of lymphovascular invasion) who have non-palpable ILNs on examination: dynamic sentinel lymph node biopsy (DSLNB) or modified or superficial ILND (sILND) (24).

Modified ILND (mILND) aims to decrease the morbidity related to radical ILND by limiting surgical dissection to the superficial lymph node packet which is anterior to the fascia lata. Several modifications have been employed to minimise morbidity. These include a smaller skin incision, preservation of the saphenous vein, conserving the area superior to the fossa ovalis and lateral to the femoral artery, and maintaining the sartorius muscle in situ (25). The inclusion of central and superior zone lymphatic tissue in the dissection is crucial, with several studies describing high false-negative rates of LNM detection because these zones were not dissected $(26,27)$. In a study by Spiess et al., 31 patients with penile SCC and non-palpable lymph nodes had sILND, with no recurrences demonstrated at up to 3-year follow-up in patients with no metastatic disease found on mILND (28). In comparison with standard radical ILND, mILND causes less morbidity without compromising oncological outcome, although the false negative rate of mILND in comparison with radical ILND has not being directly compared in a randomised trial for $\mathrm{cN} 0$ disease. The rate of major complications after mILND has been reported to be approximately $27 \%$ (29).

sILND is usually performed via a $6-8 \mathrm{~cm}$ incision parallel and inferior to the inguinal crease, and involves dissection of flaps deep to Scarpa's fascia. In sILND, the fascia lata is kept intact, with all nodal tissue superficial to it removed. When compared to mILND, the boundaries of dissection are extended medially and laterally to include the midpoint of the adductor longus muscle and the lateral boundary of the midpoint of the sartorius muscle (30). Similar to mILND, the long saphenous vein is preserved. If superficial nodes are negative, then deep or PLN dissection are not required as metastasis occurs in a stepwise manner, from superficial to deep lymph nodes.

DSLNB aims to reduce morbidity while maintaining an acceptable false negative rate. This is achieved by combining lymphoscintigraphy with technetium 99 and blue dye, subcutaneously injected around the primary tumour or scar, to define the draining lymph nodes. It has a reported false negative rate of as low as $4.8 \%$ (16), with a reported sensitivity of $88 \%$, which improved to $90 \%$, with the addition of patent blue dye, in pooled meta-analysis (31).
Previous static techniques without lymphoscintigraphy or visual identification of the sentinel nodes, as described Cabanas et al. in 1977 (32) based on lymphangiograms, has been associated with unacceptably high false-negative rates and have been deserted. Nonetheless, there is a significant learning curve associated with DSLNB, estimated at about 25 procedures (33). Given the rarity of this disease, proficiency in this technique is a challenge outside quaternary treatment settings.

All described methods of invasive lymph node staging have the potential to fail to detect micro-metastatic disease, and may lead to regional nodal recurrence (18). Ipsilateral radical inguinal lymphadenectomy is indicated if lymph node metastasis is detected. In addition, advances in minimally invasive surgery, such as endoscopic and roboticassisted techniques, have aided further attempts to reduce morbidity of inguinal lymphadenectomy Several studies have compared open inguinal lymphadenectomy with endoscopy or robotic ILND. Singh et al. (34) demonstrated that robotic lymphadenectomy produced comparable lymph node yield to open ILND (median node yield 13 vs. 12.5) with a lower major complication rate, hospital stay and drain time. In this series, no disease recurrences were reported at up to 9 months follow-up. This is supported by several smaller studies $(35,36)$ and a recent systematic review $(37)$.

\section{Palpable ILNs}

Palpable lymphadenopathy must be evaluated promptly, as it is highly suggestive of lymph node metastasis. On examination, the number of palpable nodes, their character and whether they are fixed or mobile should be noted. Imaging of the inguinal region does not alter management as palpably enlarged groin lymph node is an indication for surgical resection, however radiological evaluation with CT or MRI is indicated to detect distant metastasis. In clinically uncertain cases, fine needle aspiration cytology is an option which may guide treatment choice (38). The concept that these nodes may be reactive in the presence of infection and that antibiotic treatment should be trialled first is unsupported and may delay definitive treatment.

Patients with fixed or bulky nodal disease diagnosed on clinical exam (cN3) indicates extensive lymphatic metastatic disease with a poor prognosis and require staging by CT for systemic disease. In this setting, the use of neoadjuvant chemotherapy followed by consolidative radical surgery is currently recommended by EAU and NCCN guidelines as primary surgery alone has a low probability of cure 
$(24,39)$. Patients who have a good response to neoadjuvant chemotherapy and undergo surgery after receiving chemotherapy have been described to have long-term survival in $37 \%$ of cases (40). Currently, no tumour markers available for detection of penile cancer.

Radical inguinal lymphadenectomy is indicated if ILN metastases are detected (24). Radical ILND described by Daseler et al. in 1948 involves surgical excision of the both the superficial and deep ILN groups. It involves an incision parallel and approximately $2 \mathrm{~cm}$ inferior to the inguinal ligament, with skin flaps extending to $3 \mathrm{~cm}$ superior to the inguinal ligament, and inferiorly to the lower margin of the dissection. The flaps are developed leaving Scarpa's fascia with the flap to reduce the rate of necrosis. The boundaries of the dissection for radical ILND are: superiorly from the margin of external ring to the anterior superior iliac spine (ASIS), laterally from ASIS inferiorly $20 \mathrm{~cm}$ and medially to a line $15 \mathrm{~cm}$ downwards from the pubic tubercle. The long saphenous vein is typically ligated and transected at the apex of the femoral triangle (23). To prevent injury to the femoral nerve, the tissue adjacent to the lateral surface of the femoral artery is preserved. In most cases, the nodal tissue anterior to the femoral neurovascular structures is removed and the Sartorius muscle is transposed to protection the femoral neurovascular structures. On closure, excess skin may be excised and closure of subcutaneous tissue may assist in the reduction of the incidence of post-operative seroma.

Therapeutic radical ILND can dramatically improve patient survival and must not be underutilised for because of the potential associated morbidity. Historically, the complication rate of radical ILND has been reported between 50-70\%, with modern series suggesting lower complication rates of approximately $42 \%$ to $57 \%$ for patients who underwent ILND (29). The significant morbidity associated with radical surgery is largely due to diminished lymph return from the lower limb and scrotum, and is increased in patients with risk factors such as increased body mass index (BMI) (41). Complications which are commonly reported include lymphocele, lymphoedema, haematoma, wound infection and dehiscence, deep vein thrombosis (DVT) and necrosis. Intraoperative factors that aim to reduce post-operative morbidity include avoiding $\mathrm{S}$-shaped and $\mathrm{T}$-shaped incisions, meticulous tissue handling, preserving the saphenous vein and ligation of lymphatic vessels, whose walls do not contain smooth muscle and thus cannot be secured reliably by electrocautery alone, reduced use of metallic surgical clips and use of myocutaneous flaps where appropriate (42). Post- operative measures to reduce morbidity include the use of compression devices, stockings, inguinal vacuum suction or pressure dressings, early mobilisation, prophylactic thromboembolic prevention strategies and prophylactic antibiotics (43). Currently, there is no consensus recommendations concerning post-operative drain tube management. Spiess et al. recommend continued drainage until drain output is less than $30 \mathrm{~mL}$ per 24 hours (29). In addition, the median number of LNs resected was an independent predictor of major complication (29). A particularly distressing complication of ILND is longterm lymphoedema, with the number of patients who have lymphoedema post-operatively between $15 \%$ to $57 \%$ (29).

\section{Pelvic Iymphadenectomy}

Ipsilateral pelvic lymphadenectomy is only indicated when metastatic disease involves greater than 2 ILNs on one side and/or in the presence of extracapsular extension, as there is no direct lymphatic drainage to PLNs (24). Factors predicting PLN involvement include the number of inguinal nodes involved, the size of involved inguinal nodes, the presence of nodal extracapsular extension, aggressive histological subtype or p53 mutations. Patients with pelvic lymphadenopathy have a poorer prognosis than patients with only ILN involvement, with a 5 -year cancer specific survival of $71 \%$ vs. $33 \%$ (44). Pelvic lymphadenectomy can be performed concurrently with inguinal lymphadenectomy or as a subsequent operation. A pelvic lymphadenectomy for penile cancer aims to clear the obturator, external iliac and internal iliac nodal packets (45). The boundaries of pelvic lymphadenectomy are the genitofemoral nerve laterally, the obturator nerve medially and superiorly to the common iliac vessels. The inferior boundary of the pelvic node dissection is the superior aspect of the inguinal lymphadenectomy as the lymphatics drain into the pelvis. Bilateral pelvic lymphadenectomy is indicated if bilateral ILN involvement is suspected however spread from ILNs to the contralateral hemipelvis has not been described and is not warranted for ipsilateral inguinal disease. Pelvic lymphadenectomy may be performed as an open procedure via a lower midline or muscle splitting incision, or as a minimally invasive procedure robotically or laparoscopically according to surgeon preference and patient selection.

\section{Salvage lymphadenectomy}

Salvage inguinal lymphadenectomy may be undertaken with 
similar resection margins unless there is inguinal recurrence outside of these boundaries, however improvement in overall survival is associated with significant morbidity. A small retrospective review of 20 patients who had salvage inguinal lymphadenectomy following recurrence after primary ILND reported a complication rate of 11 of 20 patients and time to recurrence of 7.7 months and median survival of 10.1 months, with no evidence of disease in 9 patients (follow-up median 12 months, range, 7.1-70.1 months) (46).

\section{Systemic therapy}

Adjuvant chemotherapy, particularly triple combination (vincristine, bleomycin and methotrexate), after radical ILND in patients with node-positive disease has been described in small and heterogenous series. It is recommended when feasible and there is curative intent in patients with pT2 and pT3 disease (24). Improved disease-free survival has been demonstrated in patients with pathologically node-positive disease who underwent combination adjuvant therapy (vincristine, bleomycin and methotrexate) in one small retrospective study, with those patients receiving adjuvant chemotherapy having $84 \%$ disease free survival compared with $39 \%$ in those patients without adjuvant chemotherapy (40). More recently, the use of cisplatin and 5-FU or cisplatin and 5-FU plus docetaxel or paclitaxel has also shown improved outcomes $(47,48)$.

There is limited data currently available on the use of neoadjuvant chemotherapy prior to ILND, with guidelines based mainly on retrospective studies. However, neoadjuvant chemotherapy is recommended by EAU guidelines in patients with fixed or relapsed ILN metastases to facilitate surgical treatment, with the added benefit of allowing for early treatment of systemic disease (24). Fine needle aspiration or excisional biopsy should be used to confirm the presence of nodal metastasis. In patients with a complete response post neoadjuvant chemotherapy, surgical treatment is recommended with good outcomes. Proposed similarities in the pathophysiology of penile and head and neck SCC supported the evaluation of taxanes in penile SCC. The combination of 5-FU and cisplatin in addition to a taxane chemotherapeutic agent has been used in both adjuvant and neoadjuvant settings (49). Current protocols for neoadjuvant chemotherapy are cisplatin-based, with the most common regimen based on a phase II clinical trial which assessed treatment with 4 cycles of neoadjuvant paclitaxel, cisplatin and ifosfamide (TIP) with an objective response stated in 15 out of 30 patients including 3 patients who had pathologically complete remissions. The estimated median time to progression was 8.1 months, and the median overall survival being 17.1 months (50). Regimens are based upon the patient's ability to tolerate cisplatin-based regimen and institutional experience.

There is minimal data supporting the possible benefit of chemoradiotherapy combined with lymphadenectomy in penile cancer, and its use should be reserved for the clinical trial setting (51).

\section{Future directions}

Due to the rarity of the penile SCC and the lack of data relating to the molecular drivers in penile cancer in part due to lack of robust pre-clinical models, the development of novel therapeutic agents has stalled. In addition, most clinical studies are based on small, single centre series. Multidisciplinary approaches may be effective in a select patient population, there remains a considerable absence of therapeutic choices for the vast majority, particularly targeted therapies, for patients who have failed chemotherapy. In addition, there is minimal data relating to second line chemotherapy options in patients with penile SCC.

Immunotherapy is quickly changing our current model of cancer therapy, provides a useful addition to the available treatment options (52). Immunotherapy has been successfully applied in a small number of highly immunogenic tumours with varying results, ranging from no response to long-term cure. The treatment of immunogenic solid tumours, such as melanoma and renal cell carcinoma, has being significantly advanced by the recent discovery of the pivotal role of CTLA-4 and PD-1 immune checkpoint receptors, whose discoveries were awarded the 2018 Nobel Prize in Physiology or Medicine. These immunogenic tumours can be easily recognised by the immune system, and respond to removal of immune checkpoint 'breaks' which has generated a new 'fourth pillar' for cancer treatment leading to the routine clinical use of immunotherapy. Investigating the role of immunotherapy in a wider variety of tumours and enhancing the efficacy and durability of their action in the current responsive cancer types are likely to be the main advances.

Immune checkpoints, the body's natural breaks to reduce the immune response and prevent autoimmunity, form therapeutic targets and include programmed cell death (PD)-1, and cytotoxic T lymphocyte associated antigen (CTLA)-4 therapeutic targets. Immunotherapies targeting 
the PD-1/PD-1 ligand (PD-L1) axis demonstrates success in treating other urological malignancies (53). Translational studies demonstrate high rates of infiltrating immune cells and PD-L1 positivity in penile SCC, suggesting immunotherapy may be beneficial (54). However, investigating this hypothesis is challenging due to low numbers of patients to include in clinical trials. Currently there is a phase II study of pembrolizumab in patients with advanced penile SCC who have undergone prior chemotherapy, and have locally advanced unresectable or metastatic disease and have evidence of progressive disease after 1 or more chemotherapy regimen (NCT02837042) (55). Penile cancer has also been included in other basket clinical trials researching immunotherapy in patients with rare tumours or virusassociated cancer $(55,56)$. It is important that clinicians are aware of the potential to enroll patients with rare and aggressive diseases, such as penile cancer, in clinical trials. International effort is required to recruit patients to these trials as single centres or even nation-wide recruitment are unlikely to accrue large enough numbers to demonstrate significant results in this rare disease. The International Penile Advanced Cancer Trial (INPACT) is an international phase III trial, with a Bayesian design, of patients with penile cancer who have ILN metastasis. INPACT aims to determine if there is a role for neoadjuvant therapy in this patient group, and if prophylactic PLND improves survival in patients at high risk of recurrence following ILND. INPACT highlights the importance of international collaboration in providing evidence-based answers in penile cancer.

In addition to international collaboration, the centralisation of treatment of penile cancer to quaternary referral centres can improve outcomes and facilitate standardised investigations, treatment, follow up and offer platforms for enrolment into clinical trials where targeted chemotherapeutic and immunotherapeutic regimes can be trialled. Treatment received in centres with a special interest in penile cancer have been demonstrated to improve oncological outcomes of patients with penile cancer (57-59).

\section{Conclusions}

Further investigation concerning the ideal management of ILN disease in patients with penile cancer is needed, with lymphadenectomy remaining a key part of the treatment of penile cancer. International collaborative trials, such as INPACT, are essential in providing evidence on the optimal management of lymph nodes in this rare disease. DSLNB and superficial LND play a role in minimising treatment related morbidity and optimising oncological outcomes. In patients with advanced penile SCC, immunotherapy has shown early potential to provide benefit, however larger and more robust trials are needed to solidify the role and timing of these drugs.

\section{Acknowledgments}

Funding: None.

\section{Footnote}

Provenance and Peer Review: This article was commissioned by the Guest Editor (Shomik Sengupta) for the series "Surgery for Urologic Cancers" published in Translational Andrology and Urology. The article has undergone external peer review.

Conflicts of Interest: The series "Surgery for Urologic Cancers" was commissioned by the editorial office without any funding or sponsorship. The authors have no conflicts of interest to declare.

Ethical Statement: The authors are accountable for all aspects of the work in ensuring that questions related to the accuracy or integrity of any part of the work are appropriately investigated and resolved.

Open Access Statement: This is an Open Access article distributed in accordance with the Creative Commons Attribution-NonCommercial-NoDerivs 4.0 International License (CC BY-NC-ND 4.0), which permits the noncommercial replication and distribution of the article with the strict proviso that no changes or edits are made and the original work is properly cited (including links to both the formal publication through the relevant DOI and the license). See: https://creativecommons.org/licenses/by-nc-nd/4.0/.

\section{References}

1. Kieffer JM, Djajadiningrat RS, van Muilekom EA, et al. Quality of life for patients treated for penile cancer. J Urol 2014;192:1105-10.

2. Audenet F, Sfakianos JP. Psychosocial impact of penile carcinoma. Transl Androl Urol 2017;6:874-8.

3. Backes DM, Kurman RJ, Pimenta JM, et al. Systematic 
review of human papillomavirus prevalence in invasive penile cancer. Cancer Causes Control 2009;20:449-57.

4. Christodoulidou M, Sahdev V, Houssein S, et al. Epidemiology of penile cancer. Curr Probl Cancer 2015;39:126-36.

5. Cubilla AL, Velazquez EF, Amin MB, et al. The World Health Organisation 2016 classification of penile carcinomas: a review and update from the International Society of Urological Pathology expert-driven recommendations. Histopathology 2018;72:893-904.

6. Skeppner E, Andersson SO, Johansson JE, et al. Initial symptoms and delay in patients with penile carcinoma. Scand J Urol Nephrol 2012;46:319-25.

7. Kirrander P, Sherif A, Friedrich B, et al. Swedish National Penile Cancer Register: incidence, tumour characteristics, management and survival. BJU Int 2016;117:287-92.

8. Pow-Sang MR, Ferreira U, Pow-Sang JM, et al. Epidemiology and natural history of penile cancer. Urology 2010;76:S2-6.

9. Leijte JA, Kerst JM, Bais E, et al. Neoadjuvant chemotherapy in advanced penile carcinoma. Eur Urol 2007;52:488-94.

10. Srinivas V, Morse MJ, Herr HW, et al. Penile cancer: relation of extent of nodal metastasis to survival. J Urol 1987;137:880-2.

11. Leijte JA, Kirrander P, Antonini N, et al. Recurrence patterns of squamous cell carcinoma of the penis: recommendations for follow-up based on a two-centre analysis of 700 patients. Eur Urol 2008;54:161-8.

12. Kroon BK, Horenblas S, Lont AP, et al. Patients with penile carcinoma benefit from immediate resection of clinically occult lymph node metastases. J Urol 2005;173:816-9.

13. Verhoeven RH, Janssen-Heijnen ML, Saum KU, et al. Population-based survival of penile cancer patients in Europe and the United States of America: No improvement since 1990. Eur J Cancer 2013;49:1414-21.

14. Johnson TV, Hsiao W, Delman KA, et al. Extensive inguinal lymphadenectomy improves overall 5-year survival in penile cancer patients: results from the Surveillance, Epidemiology, and End Results program. Cancer 2010;116:2960-6.

15. Hegarty PK, Kayes O, Freeman A, et al. A prospective study of 100 cases of penile cancer managed according to European Association of Urology guidelines. BJU Int 2006;98:526-31.

16. Mehralivand S, van der Poel H, Winter A, et al. Sentinel lymph node imaging in urologic oncology. Transl Androl
Urol 2018;7:887-902.

17. Graafland NM, Lam W, Leijte JA, et al. Prognostic factors for occult inguinal lymph node involvement in penile carcinoma and assessment of the high-risk EAU subgroup: a two-institution analysis of 342 clinically node-negative patients. Eur Urol 2010;58:742-7.

18. Bandieramonte $\mathrm{G}$, Colecchia M, Mariani L, et al. Peniscopically controlled CO2 laser excision for conservative treatment of in situ and $\mathrm{T} 1$ penile carcinoma: report on 224 patients. Eur Urol 2008;54:875-82.

19. Slaton JW, Morgenstern N, Levy DA, et al. Tumor stage, vascular invasion and the percentage of poorly differentiated cancer: independent prognosticators for inguinal lymph node metastasis in penile squamous cancer. J Urol 2001;165:1138-42.

20. Frimberger D, Hungerhuber E, Zaak D, et al. Penile carcinoma. Is Nd:YAG laser therapy radical enough? J Urol 2002;168:2418-21; discussion 2421.

21. Meijer RP, Boon TA, van Venrooij GE, et al. Long-term follow-up after laser therapy for penile carcinoma. Urology 2007;69:759-62.

22. Dewire D, Lepor H. Anatomic considerations of the penis and its lymphatic drainage. Urol Clin North Am 1992;19:211-9.

23. Daseler EH, Anson BJ, Reimann AF. Radical excision of the inguinal and iliac lymph glands; a study based upon 450 anatomical dissections and upon supportive clinical observations. Surg Gynecol Obstet 1948;87:679-94.

24. Hakenberg OW, Minhas ES, Necchi A, et al. EAU Guidelines on Penile Cancer 2018. European Association of Urology Guidelines. 2018 Edition. Arnhem, The Netherlands: European Association of Urology Guidelines Office; 2018.

25. Catalona WJ. Modified inguinal lymphadenectomy for carcinoma of the penis with preservation of saphenous veins: technique and preliminary results. J Urol 1988;140:306-10.

26. Parra RO. Accurate staging of carcinoma of the penis in men with nonpalpable inguinal lymph nodes by modified inguinal lymphadenectomy. J Urol 1996;155:560-3.

27. Lopes A, Hidalgo GS, Kowalski LP, et al. Prognostic factors in carcinoma of the penis: multivariate analysis of 145 patients treated with amputation and lymphadenectomy. J Urol 1996;156:1637-42.

28. Spiess PE, Izawa JI, Bassett R, et al. Preoperative lymphoscintigraphy and dynamic sentinel node biopsy for staging penile cancer: results with pathological correlation. J Urol 2007;177:2157-61. 
29. Spiess PE, Hernandez MS, Pettaway CA. Contemporary inguinal lymph node dissection: minimizing complications. World J Urol 2009;27:205-12.

30. Bevan-Thomas R, Slaton JW, Pettaway CA. Contemporary morbidity from lymphadenectomy for penile squamous cell carcinoma: the M.D. Anderson Cancer Center Experience. J Urol 2002;167:1638-42.

31. Sadeghi R, Gholami H, Zakavi SR, et al. Accuracy of Sentinel Lymph Node Biopsy for Inguinal Lymph Node Staging of Penile Squamous Cell Carcinoma: Systematic Review and Meta-Analysis of the Literature. J Urol 2012;187:25-31.

32. Cabanas RM. An approach for the treatment of penile carcinoma. Cancer 1977;39:456-66.

33. Tanis PJ, Nieweg OE, Hart AA, et al. The illusion of the learning phase for lymphatic mapping. Ann Surg Oncol 2002;9:142-7.

34. Singh A, Jaipuria J, Goel A, et al. Comparing Outcomes of Robotic and Open Inguinal Lymph Node Dissection in Patients with Carcinoma of the Penis. J Urol 2018;199:1518-25.

35. Russell CM, Salami SS, Niemann A, et al. Minimally Invasive Inguinal Lymphadenectomy in the Management of Penile Carcinoma. Urology 2017;106:113-8.

36. Master VA, Jafri SM, Moses KA, et al. Minimally Invasive Inguinal Lymphadenectomy via Endoscopic Groin Dissection: Comprehensive Assessment of Immediate and Long-Term Complications. J Urol 2012;188:1176-80.

37. Gkegkes ID, Minis EE, Iavazzo C. Robotic-assisted inguinal lymphadenectomy: a systematic review. J Robot Surg 2019;13:1-8.

38. Saisorn I, Lawrentschuk N, Leewansangtong S, et al. Fine-needle aspiration cytology predicts inguinal lymph node metastasis without antibiotic pretreatment in penile carcinoma. BJU Int 2006;97:1225-8.

39. Clark PE, Spiess PE, Agarwal N, et al. Penile cancer: Clinical Practice Guidelines in Oncology. J Natl Compr Canc Netw 2013;11:594-615.

40. Pizzocaro G, Piva L. Adjuvant and neoadjuvant vincristine, bleomycin, and methotrexate for inguinal metastases from squamous cell carcinoma of the penis. Acta Oncol 1988;27:823-4.

41. Bouchot O, Rigaud J, Maillet F, et al. Morbidity of inguinal lymphadenectomy for invasive penile carcinoma. Eur Urol 2004;45:761-5; discussion 765-6.

42. Protzel C, Alcaraz A, Horenblas S, et al. Lymphadenectomy in the surgical management of penile cancer. Eur Urol 2009;55:1075-88.
43. La-Touche S, Ayres B, Lam W, et al. Trial of ligation versus coagulation of lymphatics in dynamic inguinal sentinel lymph node biopsy for staging of squamous cell carcinoma of the penis. Ann R Coll Surg Engl 2012;94:344-6.

44. Tobias-Machado M, Tavares A, Ornellas AA, et al. Video endoscopic inguinal lymphadenectomy: a new minimally invasive procedure for radical management of inguinal nodes in patients with penile squamous cell carcinoma. J Urol 2007;177:953-7; discussion 958.

45. Azizi M, Chipollini J, Peyton CC, et al. Current controversies and developments on the role of lymphadenectomy for penile cancer. Urol Oncol 2019;37:201-8.

46. Baumgarten AS, Alhammali E, Hakky TS, et al. Salvage surgical resection for isolated locally recurrent inguinal lymph node metastasis of penile cancer: international study collaboration. J Urol 2014;192:760-4.

47. Nicolai N, Sangalli LM, Necchi A, et al. A Combination of Cisplatin and 5-Fluorouracil With a Taxane in Patients Who Underwent Lymph Node Dissection for Nodal Metastases From Squamous Cell Carcinoma of the Penis: Treatment Outcome and Survival Analyses in Neoadjuvant and Adjuvant Settings. Clin Genitourin Cancer 2016;14:323-30.

48. Noronha V, Patil V, Ostwal V, et al. Role of paclitaxel and platinum-based adjuvant chemotherapy in high-risk penile cancer. Urol Ann 2012;4:150-3.

49. Giannatempo P, Paganoni A, Sangalli L, et al. Survival analyses of adjuvant or neoadjuvant combination of a taxane plus cisplatin and 5-fluorouracil (T-PF) in patients with bulky nodal metastases from squamous cell carcinoma of the penis (PSCC): Results of a single high-volume center. J Clin Oncol 2014;32:377.

50. Pagliaro LC, Williams DL, Daliani D, et al. Neoadjuvant paclitaxel, ifosfamide, and cisplatin chemotherapy for metastatic penile cancer: a phase II study. J Clin Oncol 2010;28:3851-7.

51. Eliason M, Bowen G, Bowen A, et al. Primary treatment of verrucous carcinoma of the penis with fluorouracil, cisdiamino-dichloro-platinum, and radiation therapy. Arch Dermatol 2009;145:950-2.

52. Pham T, Roth S, Kong J, et al. An Update on Immunotherapy for Solid Tumors: A Review. Ann Surg Oncol 2018;25:3404-12.

53. Motzer RJ, Escudier B, McDermott DF, et al. Nivolumab versus Everolimus in Advanced Renal-Cell Carcinoma. N Engl J Med 2015;373:1803-13. 
54. Ottenhof SR, Djajadiningrat RS, de Jong J, et al. Expression of Programmed Death Ligand 1 in Penile Cancer is of Prognostic Value and Associated with HPV Status. J Urol 2017;197:690-7.

55. Trial of Pembrolizumab for Advanced Penile Squamous Cell Carcinoma (NCT02837042). Available online: https://clinicaltrials.gov/ct2/show/NCT02837042?term= $\% 22$ penile+cancer\%22\&recr=Open\&rank $=5$

56. Nivolumab Combined With Ipilimumab for Patients With Advanced Rare Genitourinary Tumors. Available online: https://www.centerwatch.com/clinical-trials/ listings/209144/genitourinary-cancer-nivolumab-

Cite this article as: Teh J, Duncan C, Qu L, Guerra G, Narasimhan V, Pham T, Lawrentschuk N. Inguinal lymph node dissection for penile cancer: a contemporary review. Transl Androl Urol 2020;9(6):3210-3218. doi: 10.21037/tau.2019.08.37 combined-ipilimumab-patients/

57. Davis NF, Fitzgerald M, Burke JP, et al. Is there a role for the development of a supra-regional network for the management of penile cancer in the Republic of Ireland? Surgeon 2016;14:82-6.

58. Kumar P, Singh S, Goddard JC, et al. The development of a supraregional network for the management of penile cancer. Ann R Coll Surg Engl 2012;94:204-9.

59. Bayles AC, Sethia KK. The impact of Improving Outcomes Guidance on the management and outcomes of patients with carcinoma of the penis. Ann R Coll Surg Engl 2010;92:44-5. 